\title{
Research dynamic modes of stick-slip effect with the account of hereditarity
}

\author{
Roman Parovik ${ }^{1,2, \star}$ \\ ${ }^{1}$ Institute of Cosmophysical Research and Radio Wave Propagation of the Far Eastern Branch of Russian \\ Academy of Science, 684034, Kamchatskiy kray, Paratunka, Mirnaya str. 7, Russia. \\ ${ }^{2}$ Vitus Bering Kamchatka State University, 683032, Kamchatskiy kray, Petropavlovsk-Kamchatskiy, \\ Pogranichaya str. 4, Russia.
}

\begin{abstract}
In study with the help of the spectrum of maximal Lyapunov exponents, dynamic regimes of the stick-slip effect were studied with allowance effect of hereditarity. Spectrum of the Lyapunov exponents were constructed using the Wolff algorithm with Gram-Schmidt orthogonalization depending on the values of the control parametersfriction and adhesion coefficients, as well as fractional index values, which determine the heredity of the dynamical system under consideration. The existence of an area of positive values of the maximum Lyapunov exponents is shown, which indicates the presence of chaotic regimes. Oscillograms and phase trajectories are constructed.
\end{abstract}

\section{Introduction}

The stick-slip effect is investigated in tribology when the load moves on a spring along some surface of a solid body that has adhesion and dynamic friction [1]. With this movement of the load, quasiperiodic glide, its adhesion and detachment from the surface can occur, depending on the strength of the adhesion energy of the solid surface and the rigidity of the spring [2], [3].

Such motion regimes are encountered, for example, when describing the motion of a probe along the surface of a solid in an atomic force microscope in micro and nano scales [2], while studying the effect of deformation localization on the stability of friction sliding in dense amorphous materials [4], but on a macro scale the stick-slip effect is investigated, for example, in a distributed model of a drill string with Coulomb friction along a well [5], in a mechanical earthquake model [6] based on the motion of lithospheric plates and can manifest itself in electromagnetic signals lach of lithospheric origin [7].

\section{Statement of the problem}

There are various approaches to describing the stick-slip effect, for example, taking into account the atomic structure of friction bodies [8], in [9] the study of sliding objects and structures was carried out by modifying the Newmark model - a model of a rigid sliding block with Coulomb friction. This

\footnotetext{
^e-mail: parovik@ikir.ru
} 
modification somewhat weakens the rigidity of the Newmark model due to the dependence of the frictional force on its velocity (the Strybeck friction model).

We consider another approach in the approximation of equally alternating potential wells, which was proposed in [2], [3]. It is known that in classical and quantum mechanics the potential well is given by the relation:

$$
U(x)=-\frac{U_{0}}{\operatorname{ch}^{2}\left(x / x_{0}\right)},
$$

where $U_{0}$ and $x_{0}$ - depth and width of tpe hotential well.

If we expand tre Fouriwr series in (1) and differentiate with respect to the vnhiable $x$ for the periodei consirvatcve force, we obtain the folloeiag formula:

$$
F(x)=\frac{U_{0}}{x_{0}} \sum_{n=1}^{\infty} a_{n} \sin \left(n x / x_{0}\right), a_{n}=2 n \int_{0}^{1} \frac{\cos (\pi n y)}{\cosh ^{2}(\pi y)} d y .
$$

Further in [2] it was stated that for practical calculations it is sufficient to take the first seven coefficients from the formula (2), and the mathematical model of the stick-slip effect can be written in the form of the Cauchy problem:

$$
\ddot{\xi}(t)+\lambda \dot{\xi}(t)+\omega^{2} \xi(t)=b t+c \sum_{n=1}^{7} a_{n} \sin (n \xi(t)), \xi(0)=\xi_{0}, \dot{\xi}(0)=\xi_{1},
$$

where $\xi(t)=\frac{x(t)}{x_{0}}-$ displacement function, $\lambda$ - coefficient of friction, $b$ - speed of spring movement, $c=\frac{U_{0}}{x_{0}}-$ coefficient of adhesion, $\xi_{0}, \xi_{1}-$ specified constants determining initial conditions.

The mathematical model (3) describes a nonlinear oscillator with dynamic friction and external periodic action, characterizing the sticking-slip effect [3]. It should be noted that the stick-slip effect is clearly manifested in the case of a reduction in spring rigidity and the speed of its movement $b$, as well as with increasing adhesion $c$.

The time of the load in the potential well depends on the speed of the spring movement $b$. At high speeds, the loads do not practically appear, the cargo slips. The coefficient of friction $\lambda$ affects the attenuation of the cargo when it hits a potential well. Therefore, it can be noted that a significant influence on the stick-slip effect is exerted by the triple of control parameters $\lambda, b$ and $c$.

In paper, we generalize the mathematical model of the stick-slip (3) effect in the case of a hereditarity or "memory" property that occurs in hereditary mechanics [10] and can be due, for example, to the fractal properties of the surface of a solid or cargo. Following Volterra's paper [11], we can write down the following Cauchy problem:

$$
\begin{gathered}
\int_{0}^{t} K_{1}(t-\tau) \ddot{\xi}(\tau) d \tau+\lambda \int_{0}^{t} K_{2}(t-\tau) \dot{\xi}(\tau) d \tau+\omega^{2} \xi(t)= \\
=b t+c \sum_{n=1}^{7} a_{n} \sin (n \xi(t)), \xi(0)=\xi_{0}, \dot{\xi}(0)=\xi_{1}
\end{gathered}
$$

where $K_{1}(t-\tau)$ and $K_{2}(t-\tau)$ - difference kernels, which we will call memory functions.

Memory functions $K_{1}(t-\tau)$ and $K_{2}(t-\tau)$ are chosen on the basis of the structure of rubbing bodies. Of great interest, in our opinion, are the power functions of memory $K_{1}(t-\tau)$ and $K_{2}(t-\tau)$, 
and since they are the most common in nature and can to some extent describe the fractal properties of objects [12]. We choose the memory functions $K_{1}(t-\tau)$ and $K_{2}(t-\tau)$ in the form:

$$
K_{1}(t-\tau)=\frac{(t-\tau)^{1-\beta}}{\Gamma(2-\beta)}, K_{2}(t-\tau)=\frac{(t-\tau)^{-\gamma}}{\Gamma(1-\gamma)}, 1<\beta<2,0<\gamma<1,
$$

where $\Gamma(\cdot)$ - gamma function, $\beta$ and $\gamma$ - constants, associated with the fractal properties of objects or media.

The choice of functions (5) makes it possible to use the mathematical apparatus of fractional calculus. Indeed, if we substitute the relations (5) into the model equation (4) and take into account the definition of the derivative of the fractional derivative in the sense of Gerasimov-Caputo [13], [14], then finally we obtain the following non-local Cauchy problem:

$$
\partial_{0 t}^{\beta} \xi(\tau)+\lambda \partial_{0 t}^{\gamma} \xi(\tau)+\omega^{2} \xi(t)=b t+c \sum_{n=1}^{7} a_{n} \sin (n \xi(t)), \xi(0)=\xi_{0}, \dot{\xi}(0)=\xi_{1},
$$

The Cauchy problem (6) describes a fractal nonlinear oscillator and was investigated in the author's papers [15], [16]. In [17], [18], a non-local explicit finite-difference scheme was proposed and investigated, and in [15], by analogy with [19], a generalization of the model (6) was proposed in the case of order variables $\beta=\beta(t)$ and $\gamma=\gamma(t)$.

Next, we will investigate the dynamic regimes of a nonlinear fractal oscillator (6). Note that in the case of values, we get the classic stick-slip (3) effect. Therefore, we can assume that the stickslip effect, taking into account the heredity, will be affected not by three, but already by five control parameters: $\beta, \gamma, \lambda, b, c$. On the other hand, we will try to determine the conditions for the appearance of the stick-slip effect by investigating the dynamic regimes of the system (6) and bifurcation diagrams [20].

\section{Results of the study}

The investigation of dynamic regimes will be carried out with the help of constructing the spectra of maximal Lyapunov exponents for this we shall use the well-known Wolf algorithm with the GramSchmidt orthogonalization proposed in [21], as well as the nonlocal explicit finite-difference scheme for numerical calculation, investigated in the author's papers [17], [18].

Example 1. (Classic stick-slip effect). Consider the existence of the stick-slip effect as a function of the velocity of the spring $b$, described by the model (3). To do this, we select the values of the control parameters: $t \in[0,100], \lambda=0.25, \omega=1, c=50, a_{1}=0.436, a_{2}=0.344, a_{3}=0.164$, $a_{4}=0.058, a_{5}=0.021, a_{6}=0.004, a_{7}=0.003, \xi_{0}=0.2, \xi_{1}=0.3$. For a numerical scheme, we choose the number of computed nodes $N=200000$, and the step of the calculated grid tau $=0.005$. The results of the simulation are shown in Fig. 1 and Fig. 2.

In Fig. 1 that in the spectrum of maximum Lyapunov exponents positive values that correspond to the chaotic regime are observed, as well as negative ones that are responsible for the regular dynamics.

In Fig. 2 shows the following graphs: the oscillogram, the displacement velocity and the phase trajectory of the movement of the load at the speeds of the spring $b=1(b, d, f)$ and $b=2.5$ (a,c,e ).

The first case $(b=1)$ corresponds to the negative value of the Lyapunov exponent or to the regular regime. In Fig. $2 b$ shows an oscillogram of the stick-slip effect, which shows how the weight under detachment experiences damped oscillations in a potential well, the character of which depends on the coefficient of dynamic friction $\lambda$. The complete stopping of the cargo in the potential well will correspond to the case (Figure $2 \mathrm{~d}$ ). 


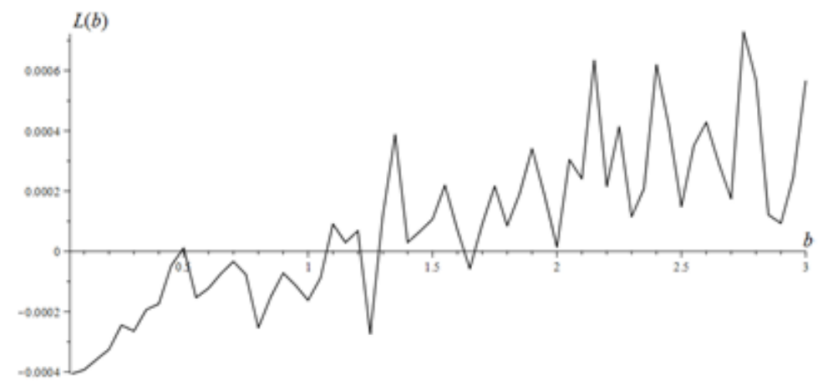

Figure 1. Spectrum of maximum Lyapunov exponents as a function of the speed of movement of the spring $b=[0.5,3]$ in increments $h=0.05$
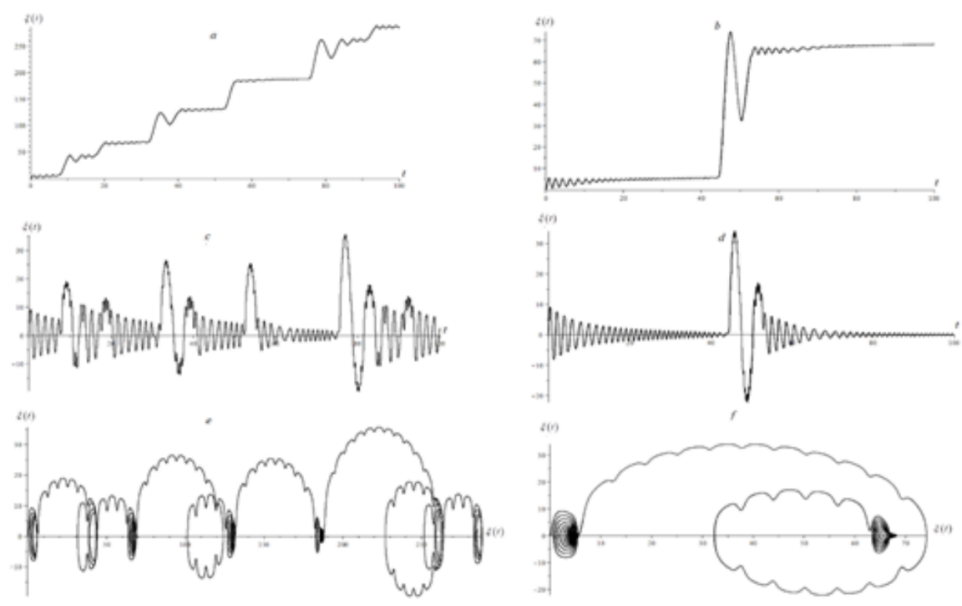

Figure 2. Oscillogram, displacement speed and phase trajectory of the load at different speed of spring movement: $b=0.25-a, c, e ; b=1-b, d, f$

The movement of the cargo to the next potential well occurs evenly - the time of finding the cargo in potential wells is the same, which is reflected in the phase trajectory (Fig. $2 f$ ).

In the case $b=0.25$, we have a positive value of the maximum Lyapunov exponent, and therefore there must be a chaotic regime. Indeed, we see in Fig. $2 a, c, e$, that the movement of cargo in potential wells is highly uneven - the time of the load in potential wells is different, also the load sometimes slips by experiencing slowly damped oscillations, so it does not stop in the potential well and hence in this case there is no stick-slip effect.

Starting from Fig. 2, we can conclude that the stick-slip effect is manifested when the speed of the spring $b$ travel decreases.

Consider the existence of the stick-slip effect as a function of the adhesion energy $c$ according to the mathematical model (3). The values of the control parameters are taken from the previous example. The spectrum of the maximum Lyapunov exponent $L$, constructed in dependence on the values $c$ of the adhesion energy of the solid surface, is shown in Fig. 3. 


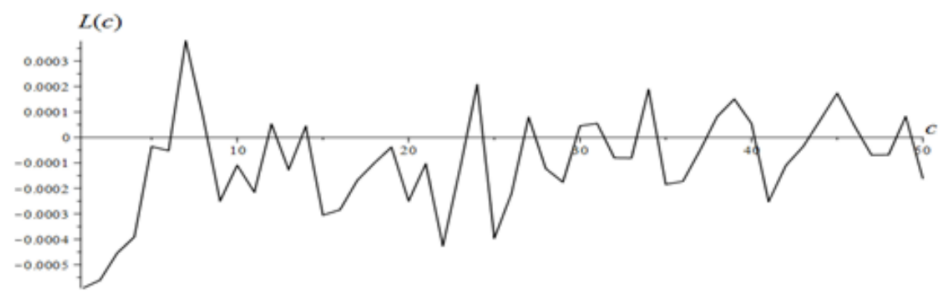

Figure 3. Spectrum of maximum Lyapunov exponents as a function of the velocity of the spring $c=[1,50]$ moving with a step $h=1$
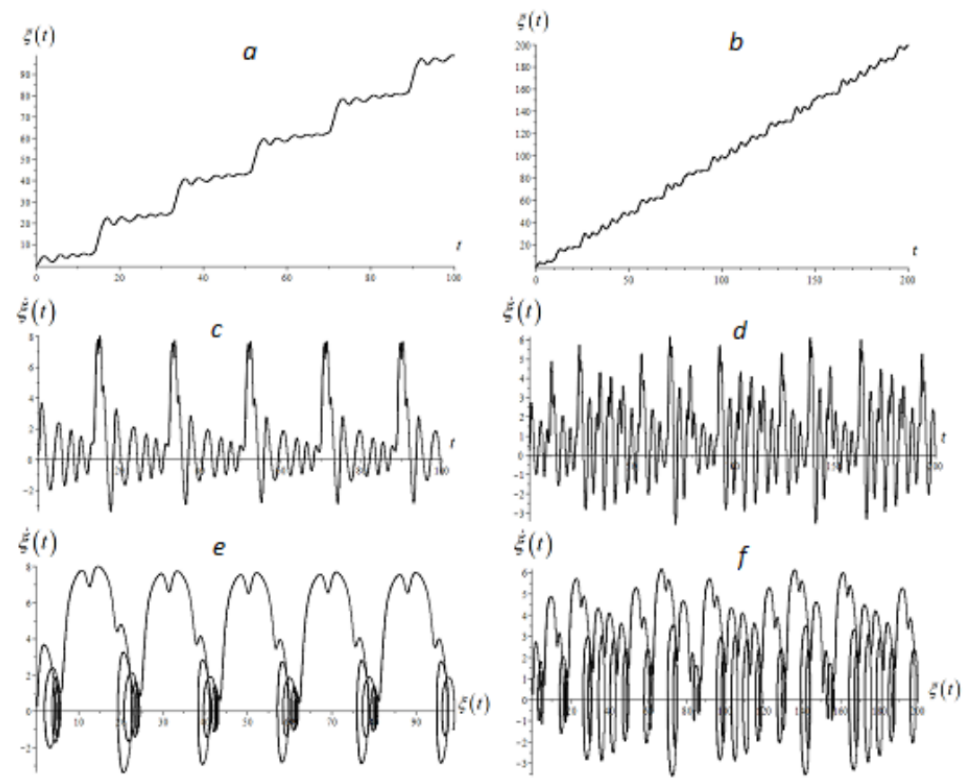

Figure 4. Oscillogram, displacement speed and phase trajectory of the load for different adhesion energy: $c=10$ $-a, c, e ; c=7-b, d, f$

In Fig. 4. Oscillograms, graphs of the speeds of cargo displacement and phase trajectories are given for surface adhesion energy values: $c=7(L>0)$ and $c=10(L<0)$. These values from the spectrum of the Lyapunov exponent (Fig. 3) were chosen as an example for demonstrating the conditions of existence and the absence of the stick-slip effect. In the first case, we've chaotic dynamics (the effect is absent), since the maximum Lyapunov exponent is positive (the effect is present), and in the second case the regular dynamics due to the negativity of the maximum Lyapunov exponent. We note that an increase in the adhesion energy of the solid surface leads to an increase in the time of the load in the potential well (Fig. 5).

From Fig. 5 that with the value of the adhesion coefficient $c=70$, the time of the load in the first potential well is about $t \approx 60 \mathrm{c}$, which is much higher $(t \approx 45 \mathrm{c})$ than $c=50$ for (Fig. $2 b)$. 


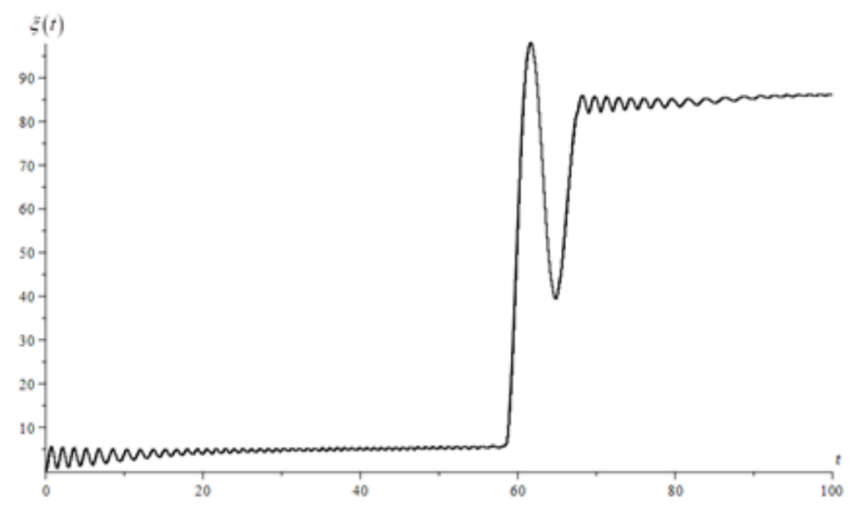

Figure 5. Oscillogram, constructed taking into account the coefficients of adhesion $c=70$, velocity of spring movement $b=1$ and dynamic friction $\lambda=0.25$

It was mentioned above that the coefficient of dynamic friction affects the nature of the damping of the oscillations.

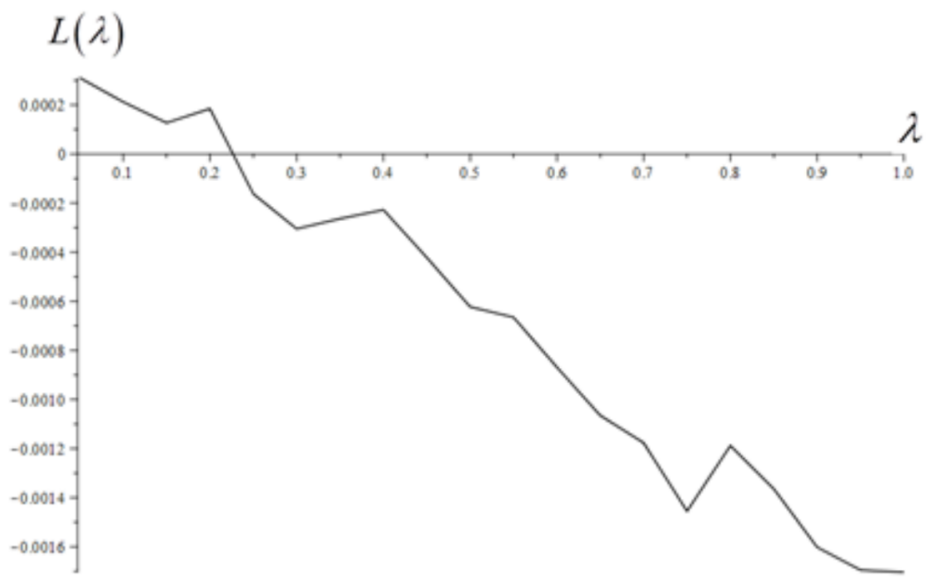

Figure 6. Spectrum of the maximum Lyapunov exponent $L(\lambda)$ as a function of the values of the coefficient of friction $\lambda \in[0,1]$ in increments $h=0.05$

From Fig. 6 we see two areas of variation of the parameter values: $\lambda, \lambda \in[0,0.225]$ and $\lambda \in$ $(0.25,1]$ where for the first region the values of the maximum exponent are positive, and for the second - negative. Therefore, the first area of variation of the parameter values is responsible for the absence of the stick-slip effect, and the second for its presence. As an example, see Fig. 7 oscillograms, displacement velocity graphs and phase trajectories of the load at friction coefficient values $\lambda: \lambda=0.1$ and $\lambda=0.5$. In the first case of Fig. 7a, $c, e$ we see that the load oscillations do not decay, and in the second Fig. $7 b, d, f$, the oscillations decay, which corresponds to the stick-slip effect. 

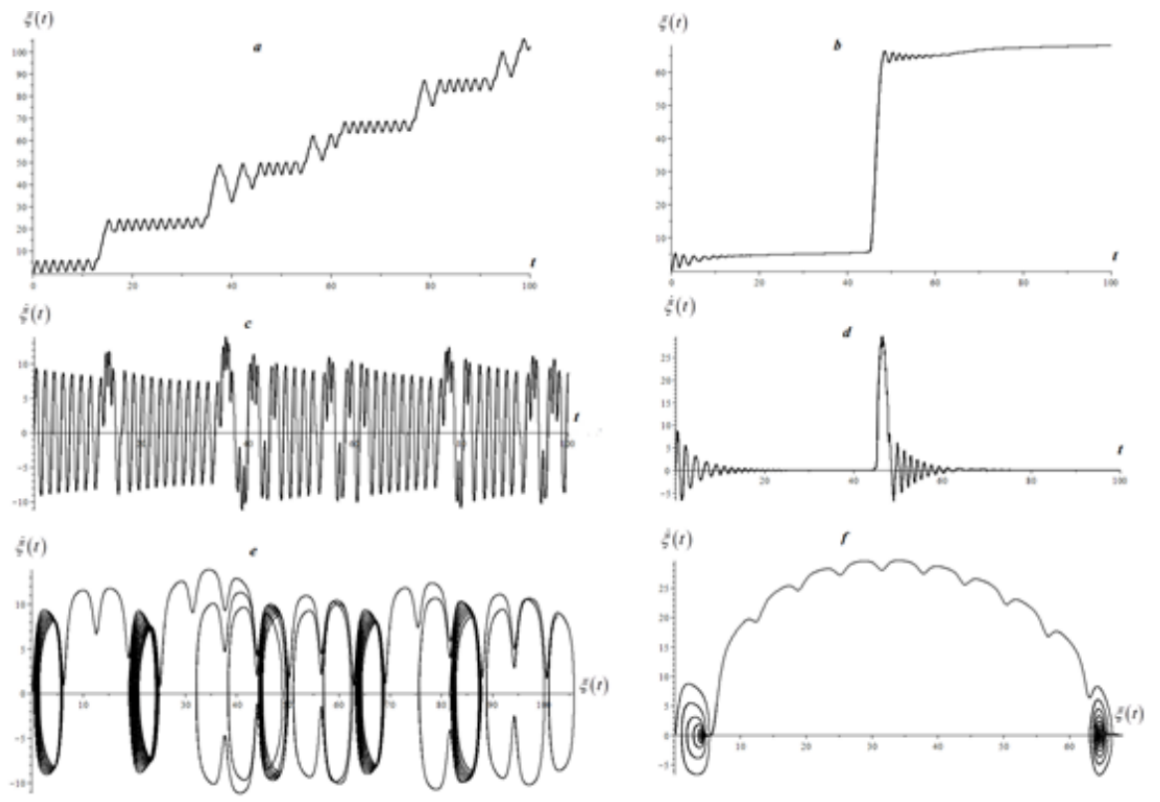

Figure 7. Oscillogram, displacement speed and phase trajectory of the load for various coefficients of dynamic friction: $\lambda=0.1-a, c, e ; \lambda=0.5-b, d, f$

We showed that the classical stick-slip effect is influenced by a triple of control parameters: $\lambda, c, b$. Consider the stick-slip effect, taking into account the heredity (control parameters $\beta$ and $\gamma$ ).

Example 2. (The stick-slip effect taking into account the heredity). The values of the basic control parameters are taken from the previous example. The number of nodes of the grid was chosen $N=8000$ to increase the counting time. We construct the spectra of Lyapunov maximum exponents with respect to the control parameters $\beta$ and $\gamma$ (Fig. 8).
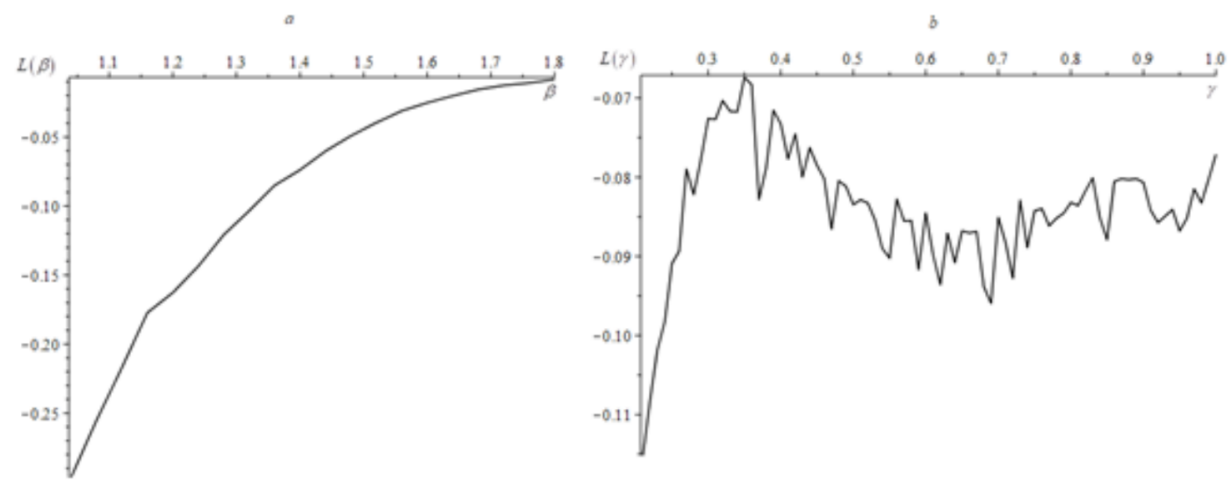

Figure 8. Spectra of maximum Lyapunov exponents: $a)-\gamma=0.8, \beta \in[1.04,1.8]$ with step $h=0.04 ; b)-$ $\beta=1.1, \gamma \in[0.21,1]$, with $h=0.1$ 
From Fig. 8 It can be seen that both spectra of the maximum Lyapunov exponent don't have positive values. This means that for this example, heredity doesn't affect the stick-slip effect. Indeed, if we recall Volterra's work [11] on the hereditarity oscillator, then in the relation for the total energy for this system an additional term appears that is responsible for the energy dissipation. Therefore, heredity for stick-slip effect will increase friction and load will not slip.

We note that the calculated curves of the Lyapunov exponents can be clarified by increasing the step of the calculated grid and the step of digitization in changing the values of the control parameters, which will lead to an increase in the estimated time.

\section{Acknowledgment}

This work was financially supported by the grant of the President of the Russian Federation No. MK-1152.2018 and the research paper "Application of fractional calculus in the theory of oscillatory processes" No AAAA-A17-117031050058-9.

\section{Conclusion}

In this work, using the Lyapunov spectral parameters, we showed that the stick-slip effect depends mainly on the three control parameters $\lambda, c, b$, the hereditarity increases friction and the effect can be pronounced. A more detailed study of the stick-slip effect can be associated with the construction of maps of dynamic regimes.

\section{References}

[1] Nachane R. P., Hussain G. F. S., Iyer K. R. Theory of stick-slip effect in friction(1998)

[2] Rekhviashvili S.SH., Razmernye yavleniya v fizike kondensirovannogo sostoyaniya $i$ nanotekhnologiyah (KBNC RAN, Nal'chik, 2014) 250

[3] Rekhviashvili S.SH., Doklady Adygskoj (CHerkesskoj) Mezhdunarodnoj akademii nauk 17, 57$56(2015)$

[4] Daub E.G., Carlson J.M., Physical Review E, 80, 066113 (2009)

[5] Ulf Jakob F. Aarsnes, Roman J., MATEC Web Conf., 148, 16002 (2018) DOI: https://doi.org/10.1051/matecconf/201814816002

[6] Scholz Ch. H., The mechanics of earthquakes and faulting (Cambridge university press, United Kingdom, 2002), 471

[7] Uvarov V.N., Vestnik KRAUNTs. Fiziko-matematicheskie nauki 14, 91-97 (2016)

[8] Nikfar F., Konstantinidis D., Journal of Engineering Mechanics 143, 04016122 (2016)

[9] Batista A.A., Carlson J.M., Physical Review E., 57, 4986-4996 (1998)

[10] Vronskij A.P., AN SSSR. Prikladnaya matematika i mekhanika 5, 31-56 (1941)

[11] Volterra V., Acta Mathematica 35, 295-356 (1912)

[12] Schroeder M., Fractals, chaos, power laws: Minutes from an infinite paradise(Freeman, 1992), 429

[13] Gerasimov A.N., AN SSSR. Prikladnaya matematika i mekhanika 2, 529-539 (1948)

[14] Caputo M., Elasticit'a e dissipazione (Bologna, Zanichelli, 1969) 150

[15] Parovik R. E3S Web of Conferences 7, 00018 (2016)

[16] Parovik R.I., Vestnik KRAUNTs. Fiziko-matematicheskie nauki 15, 30-35 (2016)

[17] Parovik R.I., Archives of control sciences 26, 429-435 (2016) 
[18] Parovik R.I., Bulletin of the south ural state university series-mathematical modelling programming \& computer software 11, 108-122 (2018)

[19] Parovik R.I., Bulletin of the south ural state university series-mathematical modelling programming \& computer software 10, 138-148 (2017)

[20] Parovik R.I., Doklady Adygskoj (CHerkesskoj) Mezhdunarodnoj akademii nauk 17, 70-77 (2015)

[21] Wolf A. et al., Physica D: Nonlinear Phenomena 16, 285-317 (1985) 